\title{
1. Einleitung
}

\author{
Hans Förster
}





\section{Der sahidische Text des Johannesevangeliums}

Die koptische Sprache stellt die jüngste Stufe des Ägyptischen dar. Zuweilen bezeichnet man das Koptische auch als die Sprache des christlichen Ägyptens, da die christlichen literarischen Texte nicht mit den älteren ägyptischen Zeichen, sondern mit griechischen Buchstaben und einer Reihe aus der demotischen Schrift, einer späten Schreibschrift der klassischen Hieroglyphenschrift, weiterentwickelter Zeichen geschrieben wurden. ${ }^{1}$ Die Übernahme des griechischen Alphabets geht mit der Übernahme zahlreicher griechischer Wörter einher, ${ }^{2}$ sodass koptische Handschriften oftmals griechisch wirken. Dieser Eindruck wird dadurch verstärkt, dass die ägyptischen Sonderzeichen kalligraphisch dem griechischen Alphabet angepasst wurden, sodass diese Buchstaben auf den ersten und oberflächlichen Blick nicht auffallen. Damit erwecken gerade die koptischen Übersetzungen des Neuen Testaments den Eindruck, bereits auf der Ebene der Schrift in einer besonderen Nähe zum Griechischen zu stehen. Aber auch abgesehen von dieser formalen Ähnlichkeit der literarischen Handschriften gilt die koptische Überlieferung des Neuen Testaments in ihrer Bedeutung für die neutestamentliche Textforschung als eine der wichtigsten altkirchlichen Bibelübersetzungen. „Die“ koptische Übersetzung des Neuen Testaments gibt es allerdings nicht. Dies ist eng mit der koptischen Sprache verbunden. Die koptische Sprache selbst teilt sich in mehrere Dialekte auf, Übersetzungen in diese einzelnen Dialekte wurden oftmals unabhängig voneinander vorgenommen. Auch mehrfache Übersetzungen eines neutestamentlichen Buchs in einen der kleineren Dialekte sind belegt. Derartige Parallelübersetzungen unterscheiden sich oftmals in grundlegender Weise voneinander. Wie eigenständig zwei voneinander unabhängige Übersetzungen in einem einzigen Dialekt sein können, zeigen die zwei von Hans Martin Schenke veröffentlichten mittelägyptischen Handschriften des Matthäusevangeliums. ${ }^{3}$ Auf der Basis eines Vergleichs der beiden stark voneinander abweichenden Zeugen dieser Übersetzung des Matthäusevangeliums kommt Schenke zu dem Schluss, dass zwei sehr verschiedene griechische Vorlagen existiert haben müssen. Diese würden die großen Unterschiede zwischen den beiden Übersetzungen am besten erklären. Es scheint jedoch, dass Schenke die Freiheiten, die sich diese Übersetzer nahmen, unterschätzt hat, sodass sich seine Hypothese nicht durchsetzen konnte. Die beiden wichtigsten und am besten bezeugten Dialekte sind das Sahidische und

1 Vgl. hierzu Förster 2011a: 29-37.

2 Vgl. hierzu Förster 2002.

3 Vgl. Schenke 2001 sowie Schenke 1981. 
das Bohairische. Bei beiden handelt es sich um koptische Literaturdialekte, wobei das Sahidische ein oberägyptischer Dialekt war, während das Bohairische ursprünglich einen unterägyptischen Dialekt darstellte. Von einer diachronen Verteilung her gesehen, liegt das Sahidische zeitlich als Literaturdialekt vor dem Bohairischen, auch wenn frühe Zeugen des Bohairischen selbstverständlich existieren. $\mathrm{Zu}$ diesen frühen Zeugen des bohairischen Dialekts gehört auch eine Papyrushandschrift, die große Teile des Johannesevangeliums im protobohairischen Dialekt bietet. ${ }^{4}$ Aufgrund der Tatsache, dass das Sahidische den früheren Literaturdialekt bildet, gibt es - im Gegensatz zum Bohairischen zahlreiche nur fragmentarisch erhaltene Handschriften des Johannesevangeliums, während im Bohairischen eine große Zahl vollständiger Handschriften erhalten ist. Der ersten kritischen Edition der sahidischen Version des Johannesevangeliums durch George Horner lagen nur Handschriftenfragmente zugrunde, die zusammen den vollständigen Text dieses Evangeliums bezeugten. ${ }^{5}$ Nach dieser Publikation wurden mehrere vollständige Handschriften des Johannesevangeliums im sahidischen Dialekt bekannt. ${ }^{6}$ In der Folge wurden mehrfach einzelne Handschriften publiziert, zuerst von Hans Quecke, der den vollständigen Text einer in Barcelona aufbewahrten Handschrift (sa 1) mit den Textvarianten einer Reihe weiterer, vollständiger Handschriften bietet. ${ }^{7}$ Vor wenigen Jahren veröffentlichte Karlheinz Schüssler den Text einer Handschrift (sa 5), die auch von Quecke für den kritischen Apparat verwendet worden war, als selbständige Edition. ${ }^{8}$ Während Quecke zumindest die Textvarianten der von ihm verwendeten Handschriften bietet, fokussiert Schüssler auf den Text einer Handschrift, ohne Abweichungen innerhalb der sahidischen Textüberlieferung $\mathrm{zu}$ thematisieren. Allerdings entscheidet sich auch Quecke für eine Handschriftenedition; das zweispaltige Textlayout ist für den Gebrauch eher nachteilig. Schüssler hingegen entscheidet sich dafür, den Text der Handschrift einmal als Fließtext und einmal als Edition unter Berücksichtigung des Textlayouts der Handschrift zu publizieren. Allerdings erhält man trotz des doppelten Abdrucks des Textes von sa 5 keinen Zugang zu den in den anderen sahidischen Hand-

4 Für die Editionen vgl. Kasser 1958 sowie Sharp 2016. Im Rahmen der Edition der hier vorgelegten sahidischen Version des Johannesevangeliums wird als Anhang auch eine Edition des proto-bohairischen Textes geboten.

5 Für die Edition vgl. Horner 1911.

6 Siehe hierzu auch Schüssler 2008: 41-62, hier 42: „We know the Gospel of John as an edited document only from one manuscript. Fortunately Hans Quecke edited the text of manuscript sa 561 very carefully and published it in 1984."

7 Quecke 1984.

8 Schüssler 2013. 
schriften gebotenen Textvarianten. Daher musste bisher - trotz aller Mängel immer noch auf Horners über einhundert Jahre alte Edition zurückgegriffen werden, vor allem wenn es um Textvarianten ging, die Schüssler überhaupt nicht berücksichtigt und die Quecke nur in Auswahl bietet.

\section{Die sahidischen Handschriften und ihre Nummern}

Es gibt eine Vielzahl koptischer Handschriften. Dies ist vor allem den für die Erhaltung der Texte wichtigen und positiven klimatischen Bedingungen in Ägypten zu verdanken. Es gibt wohl kein anderes Land der Erde, aus dem eine vergleichbare Menge an Textzeugen aus der Antike erhalten ist wie Ägypten. Das gilt dann natürlich auch für Bibelhandschriften. Für diese ist es von besonderer Bedeutung, dass die Reste verlassener Bibliotheken von Klöstern und anderen kirchlichen Einrichtungen die Jahrhunderte überdauern konnten. Umgekehrt hat diese Situation jedoch dazu geführt, dass Reisende und der Antiquitätenhandel vor allem im 19. Jahrhundert dazu beitrugen, die aus Ägypten stammenden Handschriften in die ganze Welt zu verteilen. In der Folge sind die einzelnen Blätter koptischer Bibelhandschriften oftmals auf verschiedene Bibliotheken und auch auf unterschiedliche Kontinente verteilt. ${ }^{9}$ In der Folge wurden von verschiedenen Gelehrten koptische Bibelhandschriften in den unterschiedlichsten Bibliotheken katalogisiert und diese Handschriften so der wissenschaftlichen Öffentlichkeit zugänglich gemacht. ${ }^{10}$

In der Einleitung ist deshalb auch auf das System - bzw. die Systeme - einzugehen, die für die Nummerierung sahidischer Handschriften verwendet werden. Das von Horner verwendete System hatte sich als extrem unpraktisch erwiesen und wurde deswegen in der Folge durch die im Institut für Neutestamentliche Textforschung in Münster vergebenen SMR-Nummern ersetzt. Dabei wurden auch Handschriften neu zusammengestellt und Irrtümer in den Handschriftenzusammenstellungen korrigiert. Das System basiert auf den von FranzJürgen Schmitz und Gerd Mink publizierten Forschungsarbeiten, ${ }^{11}$ die Siegfried

9 Siehe hierzu Richter 2016: 803-809, hier 804: „Because of the interest of travellers and the booming art trade since the $19^{\text {th }}$ century Coptic manuscripts today are scattered over several collections, as is the case with sa 35, which contains the Acts and the Catholic and Pauline letters. Like several other manuscripts, the leaves of this codex are preserved in museums scattered over two continents. In this case, the 36 fragmentary leaves lie in 10 museums.“

10 Für eine detailliertere Forschungsgeschichte vgl. u.a. BC 1.1: 1-2 sowie Richter 2008: 804.

11 Schmitz/Mink 1986; Schmitz/Mink 1989; Schmitz/Mink 1991; Schmitz 2003. 
Richter als Datenbank weiterführt und kontinuierlich ergänzt. ${ }^{12}$ Rund ein Jahrzehnt nach der Einführung der SMR-Nummern führte Karlheinz Schüssler ein eigenes Nummernsystem, die so genannten BC-Nummern (i.e. Biblia Coptica) ein. Dieses System konnte sich nie durchsetzen, ${ }^{13}$ auch baut Schüssler eindeutig auf den Arbeiten von Schmitz und Mink auf. ${ }^{14}$ Die BC-Nummern werden seit dem Erscheinen von Bd. 2/2 ${ }^{15}$ nicht mehr weiter vergeben. ${ }^{16}$ Auch ist eine separate Weiterarbeit an der Biblia Coptica nicht mehr vorgesehen, ${ }^{17}$ sodass alle weiteren, zu vergebenden SMR-Nummern keine Entsprechung mehr bei den BCNummern haben werden. Diese Entscheidung trägt dazu bei, die Arbeit mit koptischen Handschriften zu vereinfachen.

Bei den SMR-Nummern handelt es sich um das wissenschaftlich gebräuchliche und ältere System, das für die bisherigen Arbeiten an der Editio Critica Maior zur Anwendung kam $^{18}$ und deshalb auch im hier vorgelegten Werk zur Anwendung kommt. ${ }^{19}$ Die Bedeutung der SMR-Nummern zeigt sich auch darin, dass beispielsweise Christina Kreinecker ${ }^{20}$ oder Christian Askeland die SMR-

12 Vgl. http://intf.uni-muenster.de/smr/.

13 Hier muss erwähnt werden, dass Karlheinz Schüssler in der Einleitung zu seinem ersten Band nur kurz die von ihm verwendeten Vorarbeiten erwähnt, ohne auf die Problematik des von ihm neu eingeführten Systems zu verweisen: BC 1.1: 1: „Eine große Hilfe bieten glücklicherweise die hervorragenden Zusammenstellungen von Peter Nagel für das Alte Testament sowie die Auflistungen von Schmitz/Mink für die Evangelienhandschriften.“

14 Richter 2016: 805-806: „In his list called Biblia Coptica, Karlheinz Schüssler gathered a total of 286 Sahidic text witnesses of the New Testament. He collected information from published catalogues or articles and added at least 69 further Sahidic manuscripts containing various New Testament texts, including witnesses on ostraca and wooden panels. With regard to the remaining 217 items in Schüssler's list, the author essentially follows the work of Schmitz/ Mink, reusing data concerning composition, pagination, the sequence of the pages, aside from some individual fragments.“

15 Anzumerken ist in diesem Zusammenhang, dass die einzelnen Lieferungen der Bände nicht in ihrer numerischen Reihenfolge erschienen sind.

16 Vgl. BC 2.2.

17 Karlheinz Schüssler hat als Privatperson die Biblia Coptica-Bände herausgegeben und hätte die Arbeit noch für viele Jahre geplant gehabt. Sein Unfalltod im Jahr 2013 hat ihn aus dieser Arbeit herausgerissen (siehe Förster 2013). Nach seinem Tod hat sich niemand gefunden, der seine Arbeit weiterführen hätte können. Hier zeigt sich einmal mehr der Wert einer institutionellen Anbindung geisteswissenschaftlicher Forschung, die sicherstellt, dass derartige Arbeiten nicht ausschließlich mit einzelnen Persönlichkeiten verbunden sind und gegebenenfalls auch mit diesen ein Ende finden.

18 Vgl. Strutwolf et al. 2017b: 145-167 (4.2. Koptisch), hier 145. Durch die SMR-Datenbank erübrigt sich ein Hinweis auf Biblia Coptica. Siehe ferner Richter/Schröder 2017: 72-80.

19 Vgl. hierzu Aland 2014; Strutwolf et al. 2017 und Strutwolf et al. 2017a.

20 Vgl. Kreinecker 2008: 7. 
Nummern verwenden. ${ }^{21}$ Aufgrund der Verfügbarkeit der SMR-Nummern in einer Datenbank, die neben den notwendigen kodikologischen Informationen auch die älteren Nummern der Handschriften verzeichnet, ${ }^{22}$ konnte bei der Erstellung der Edition darauf verzichtet werden, neben den SMR-Nummern zusätzlich auch die BC-Nummern oder Horners System zu verwenden.

\section{Vorgeschichte der Edition}

Christina Kreinecker leitet mit einem Zitat von Stephen Emmel in ihre textkritischen Untersuchungen zu den koptischen Zeugen der Auferstehung ein: „Die Koptologie steckt noch in ihren Kinderschuhen“ und genau darin liegt das Problem, will man sich mit den Inhalten der Texte beschäftigen. Bis heute sind Handschriftenlisten, Sprachuntersuchungen, entsprechende Konkordanzen und Weiteres entweder im Aufbau begriffen oder greifen auf ein relativ geringes Datenmaterial in Summe zurück. ${ }^{\text {"23 }}$ Die Probleme der unterschiedlichen Handschriftenlisten und der damit verbundenen Systeme der Verzeichnung sahidischer Fragmente von Handschriften wurden bereits dargelegt. Grundsätzlich sind zahlreiche Handschriften des Johannesevangeliums im sahidischen Dialekt bekannt. Die auf oftmals viele Bibliotheken verteilten Blätter der ursprünglichen Handschrift sind in den Listen zusammengestellt. Allerdings sind die meisten der so zusammengestellten Handschriften nicht ediert. Für eine kritische Edition ist es unabdingbar, zuerst alle bekannten Handschriften zu transkribieren, um den in ihnen überlieferten Text zu kennen. Hierfür ist oftmals auf Bildmaterial zurückzugreifen, da ein Besuch aller Sammlungen, in denen Blätter einzelner sahidischer Textzeugen aufbewahrt werden, schlicht unmöglich ist.

Karlheinz Schüssler besaß nun eine umfangreiche und über viele Jahrzehnte hinweg aufgebaute Sammlung von Mikrofilmen und Fotos von koptischen Handschriften, unter denen sich selbstverständlich auch zahlreiche Abbildungen sahidischer Zeugen des Johannesevangeliums befanden. Noch zu Lebzeiten von Karlheinz Schüssler wurde in Münster und Wien mit einer Digitalisierung des Filmmaterials begonnen. Die Digitalisate befinden sich heute im Institut für

21 Askeland 2012.

22 Richter 2016: 806-807: „The online database is available on the web page of the Institute for New Testament Textual Research in Münster, and contains in version SMR 2014.1 (June 2014) information about 419 fragments. The data base incorporates the numerations of Schmitz/Mink and Biblia Coptica, the Gregory-Aland numbers, older numbers of the INTF, Horner-numbers, inventory numbers, editions and further literature.“

23 Kreinecker 2008: 2. 
Neutestamentliche Textforschung in Münster, in der Papyrussammlung der Österreichischen Nationalbibliothek und im Septuaginta Institut in Göttingen. ${ }^{24}$

Das von Karlheinz Schüssler zusammengetragene Bildmaterial bildete zusammen mit dem im Institut für Neutestamentliche Textforschung in Münster zusammengetragenen Bildmaterial die Ausgangsbasis, um die sahidische Version des Johannesevangeliums genauer zu erforschen. Das Ziel mehrerer Forschungsprojekte des österreichischen Wissenschaftsfonds (FWF-Projekte 22017 und 25082) war es, alle erreichbaren sahidischen Textzeugen des Johannesevangeliums, soweit sie heute bekannt sind, zu kollationieren. Mit dem Begriff der „erreichbaren Handschrift“ wird dabei das Problem umrissen, dass einerseits einzelne Blätter seit ihrem Bekanntwerden verschollen sind. So wurden beispielsweise auch einige Blätter im Rahmen der Kriegswirren des Zweiten Weltkriegs zerstört. Andererseits ist die Beschaffung von Bildmaterial - abhängig von den bürokratischen Hürden der einzelnen Bibliotheken - teilweise sehr einfach, teilweise ist es jedoch durchaus eine Herausforderung, alle Hürden zu überwinden. $\mathrm{Zu}$ den erreichbaren Handschriften gehörte auch der so genannte „Naqlun-Text“ (sa 19)..$^{25}$ Dieser wurde bei Ausgrabungen in Naqlun gefunden und wird im Lagerraum der Polnischen Mission mit der Nummer N.02.030 aufbewahrt. Die 2003 angekündigte „rasche editio minor“ ist bisher leider noch immer nicht erschienen. ${ }^{26}$ Gerade angesichts der wenigen vollständigen oder fast vollständigen Handschriften des Johannesevangeliums im sahidischen Dialekt hätte ein Fehlen dieser Handschrift den Wert der kritischen Edition beeinträchtigt. Vor diesem Hintergrund ist den Eigentümern der Editionsrechte für ihre Großzügigkeit an dieser Stelle ausdrücklich und herzlich zu danken: Sie haben für die vorgelegte Edition das Bildmaterial in hervorragender Qualität zur Verfügung gestellt. Deswegen war es möglich, sa 19 im kritischen Apparat zu berücksichtigen.

24 Das von Karlheinz Schüssler zusammengetragene Filmmaterial wird heute in der Papyrussammlung der Österreichischen Nationalbibliothek aufbewahrt.

25 Für einen ersten Bericht vgl. van der Vliet 2003: 172-176, hier besonders 176: „Moreover, even though the manuscript has its usual share of small copyist's errors, omissions and ad sensum additions, it appears to represent on the whole a carefully transcribed and well preserved text. $\mathrm{N}$ is therefore not only a welcome addition to the small number of complete Sahidic manuscripts of John, it also deserves to take its place in the textual history of the Sahidic NT as an independent witness on a par with A, B or M.“

26 Van der Vliet 2003: 176: „In order to make it available to the scholarly world without much delay, the rapid publication of an editio minor, more or less along the lines of H. Quecke's 'Johannesevangelium', is envisioned.“ 
Die erwähnten FWF-Projekte und damit die Vorbereitung der sahidischen Handschriften für die Verwendung der gesamten Breite der sahidischen Überlieferung im Rahmen der Editio Critica Maior begannen im Jahr 2010 als Zusammenarbeit zwischen Hans Förster und Karlheinz Schüssler. Im Verlauf der Arbeiten wurden in den Beständen von Karlheinz Schüssler fehlende Bilder bzw. Mikrofilme aus Mitteln des FWF zugekauft. Ziel der Arbeit in den erwähnten FWF-Projekten war es, die sahidische Textüberlieferung und ihre Bedeutung für die Textüberlieferung des griechischen Neuen Testaments im Rahmen der sogenannten Editio Critica Maior des Johannesevangeliums fruchtbar zu machen. Angesichts der Tatsache, dass damit Abschriften aller sahidischen Handschriften des Johannesevangeliums vorlagen, erschien es zweckmäßig, sie für eine kritische Edition zu verwenden. Dies geschah im Rahmen des FWF-Projekts 29315.

\section{Die kritische Edition}

Die Publikation einer kritischen Edition der sahidischen Version, die alle bekannten Handschriften dieser Version möglichst vollständig berücksichtigt, stellt bisher für annähernd alle Bücher des Neuen Testaments ein Desiderat der Koptologie dar. Nur einige wenige und in der sahidischen Überlieferung vergleichsweise schlecht bezeugte Briefe des Neuen Testaments wurden bisher in einer kritischen Edition vorgelegt. ${ }^{27}$ Verglichen mit der Beleglage bei den Evangelien ist die Zahl der handschriftlichen Zeugen niedrig, wie die synoptische Präsentation aller bekannten Handschriften einiger weniger Briefe durch FranzJürgen Schmitz sehr gut deutlich macht. ${ }^{28}$ Eine vergleichbare synoptische Präsentation aller bekannten Handschriften wurde für die sahidische Version des Johannesevangeliums als unzweckmäßig ausgeschlossen. Anne Boud'hors beispielsweise bezeichnet den von Franz-Jürgen Schmitz publizierten Band angesichts seines Formates als „Monster“. ${ }^{29}$ Eine solche Präsentation der Handschriften würde im Falle des Johannesevangeliums den Umfang eines normalen Buches bei Weitem sprengen. Damit wäre jedoch - allein aufgrund des in diesem Fall notwendigerweise noch größeren Formats - eine derartige Publikation

27 Für eine kritische Edition der katholischen Briefe vgl. Schüssler 1991.

28 Schmitz 2003.

29 Boud'hors 2005: 163-166, hier 163: „La première impression produite par ce livre pourra être : un monstre! 400 pages de présentation synoptique bourrées de chiffres et de sigles, 150 pages de tableaux statistiques décourageants.“ 
nur schwer zu benützen. ${ }^{30}$ Ferner ist es bei der Edition koptischer Texte aufgrund des im Vergleich mit dem Griechischen niedrigeren Normierungsgrades der Orthographie ${ }^{31}$ unüblich, graphische Varianten im Rahmen einer Normierung der Orthographie des edierten Textes zu eliminieren. Auch die Wörterbücher verzeichnen für die jeweiligen Wörter die entsprechenden graphischen Varianten. Der Effekt ist - dies ist ja bereits bei der Aufteilung der kritischen Apparate bei Horner zu sehen -, dass graphische Varianten in einem eigenen Apparat verzeichnet werden und ein weiterer Apparat die textkritisch bedeutsamen Varianten bietet. Verglichen mit Horners Edition stellt damit der kritische Apparat von Hans Quecke einen Rückschritt dar, da hier in einem einzigen Apparat unterschiedslos textkritisch bedeutsame und orthographisch auffällige Abweichungen verzeichnet werden. Eine synoptische Präsentation würde die Analyse der Überlieferung der Leserschaft überantworten. Ein derartiges Vorgehen müsste angesichts der Fülle der sahidischen Handschriften des Johannesevangeliums als benutzerfeindlich angesehen werden. Immerhin wurde die Arbeit an der kritischen Edition innerhalb eines auf drei Jahre angelegten FWFProjekts durchgeführt, was deutlich zeigt, wie viel Zeit für die Auswertung der Handschriften und die Erstellung eines so umfangreichen kritischen Apparats benötigt wird.

Das wichtigste Ziel der kritischen Edition ist es, der Leserschaft einen möglichst einfachen Zugang zur Textüberlieferung mit allen Varianten zu bieten. So wurde dahingehend auf Horner als Vorbild zurückgegriffen, als dass in zwei getrennten Apparaten zum einen die orthographischen Eigenheiten der Handschriften dokumentiert und zum anderen die textkritisch wichtigen Abweichungen notiert werden. Damit ist sofort ein Überblick zu gewinnen.

An dieser Stelle sind nun auch noch einige weitere Bemerkungen nötig. Grundsätzlich ist das Ziel einer jeden kritischen Textausgabe, ein möglichst

30 Boud'hors 2005: 164: „Il faut reconnaitre que le grand format du livre $(31 \times 24 \mathrm{~cm})$, par ailleurs un peu encombrant, permet une disposition aérée qui rend la consultation la moins rébarbative possible. Il est probable que, suivant ses intérêts, le lecteur y trouvera toutes sortes d'informations, ce qui es signe d'une grande richesse.“

31 Vgl. hierzu auch Schüssler 2006: 48: „It is true that in the Egyptian writing schools 'orthography' and grammar as customary to the respective place were taught, as writing exercises and textbooks show. [...] Thus e.g. there are differences from school to school. Furthermore the spelling of the occasional word changed in the course of time, e.g. in the case of xekac or $x$ ekdac. In dictionaries we find both versions of spelling but $x \in$ ekdac is the predominant form in the oldest manuscripts whereas in more recent manuscripts the simplified form is used. This example alone shows that differences in spelling cannot be regarded as mistakes, but rather that the evolution of the language has to be taken into account.“ 
frühes Stadium bzw. den Archetyp eines Textes aufgrund stemmatologischer Untersuchungen aufzuzeigen und als Edition zugrunde zu legen. Die handschriftlich bezeugten textlichen Abweichungen vom Archetyp sind entsprechend im kritischen Apparat zu berücksichtigen. Ein derartiges Vorgehen bzw. der Versuch, einen Archetyp zu identifizieren, steht bei der sahidischen Überlieferung des Johannesevangeliums vor großen methodischen Problemen. Grundsätzlich sind die Probleme mit denen vergleichbar, vor denen auch eine kritische Edition des griechischen Textes steht. Bei der Überlieferung des neutestamentlichen Textes handelt es sich um eine vielfach kontaminierte Textüberlieferung. ${ }^{32}$ Die Kontamination der Überlieferung ist im Falle der sahidischen Überlieferung darauf zurückzuführen, dass einerseits wohl mindestens eine Revision des sahidischen Textes des Johannesevangeliums gegen eine griechische Handschrift stattgefunden hat. Dies hat zur Folge, dass die sahidische Überlieferung an textkritisch bedeutsamen Stellen - es sei beispielsweise auf Joh 1,39 verwiesen - zwei textkritisch wichtige Varianten bezeugt. ${ }^{33}$ Fraglich muss bleiben, ob dabei die Handschrift, die formal in einigen Fällen näher am griechischen Text ist, was Satzstellung und vergleichbare Aspekte betrifft (der wichtigste Vertreter dieser formal näher am griechischen Text befindlichen Handschriften ist sa 1), auch tatsächlich den „ältesten Text“ bietet. ${ }^{34}$ Immerhin

32 Dies hat große Bedeutung für die Ermittlung eines Stemmas; vgl. Maas 1960: 6: „Voraussetzung des Folgenden ist, daß die seit der Hauptspaltung geschehenen Abschriften immer nur je eine Vorlage wiedergeben (d. h. daß kein Schreiber mehrere Vorlagen ineinanderarbeitet, 'kontaminiert'), daß dagegen jeder Schreiber bewußt oder unbewußt von seiner Vorlage abweicht (Sonderfehler begeht).“

33 Gegen Schüssler (Schüssler 2006: 55). Er verweist auf den Wechsel zwischen Futur und

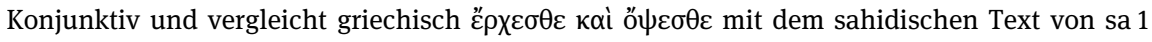

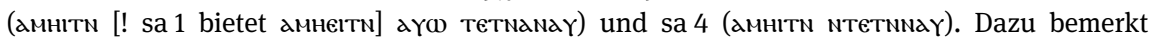
Schüssler: ,[...] however dependent on the understanding of the form of ö $\psi \varepsilon \sigma \theta \varepsilon$ : this may be an imperative as in $\varepsilon^{\prime} \rho \varepsilon \sigma \theta \varepsilon$; the corresponding form in Coptic would be the conjunctive NTETnNaY, or this is an infinitive future active, where the corresponding Coptic form would be the future in aүш тетманаү." Das finite Verb ö $\psi \varepsilon \sigma \theta \varepsilon$ kann keinesfalls als Infinitiv des Futurs angesehen werden. Es ist vielmehr die 2. Person Plural des medialen Futurs. Der Impe-

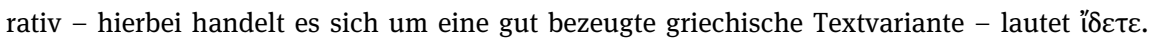
Hier liegen also zwei unterschiedliche griechische Ausgangstexte der koptischen Überlieferung zugrunde. Der griechischen Form ő $\psi \varepsilon \sigma \theta \varepsilon$ liegt - gegen Schüssler - einzig und allein die sahidische Variante aүळ тетманаץ zugrunde. Vgl. auch Boud'hors 2005: 164: „[...] décider si des tournures coptes différentes sont ou non des traductions d'un même texte grec nécessite en effet des prises de position d'ordre linguistique.“ Siehe auch Maas 1960:- 13: „Bei zweigespaltener Überlieferung führt die recensio oft [...] auf zwei Varianten.“

34 So argumentiert Schüssler 2008: 61: „Comparison with the Greek texts shows that one of the Sahidic texts, namely sa 561 attempts to follow the word order of the Greek 'Vorlage' as 
- dies zeigt ja die eingangs erwähnte von Hans Martin Schenke angestoßene Diskussion - scheinen gerade „freie“ oder sogar „wilde“ Übersetzungen gegenüber besonders textgetreuen Übertragungen ein höheres Alter zu besitzen. ${ }^{35}$ Auch müsste in diesem Fall erklärt werden, warum allein sa 1 Joh 16,15 auslässt. Falls es sich bei sa 1 um die älteste Form des sahidischen Textes handeln sollte, muss unverständlich bleiben, warum dieser Fehler - der dann ja auf eine griechische Textvariante zurückginge - nicht in der sahidischen Überlieferung erhalten blieb. Andererseits scheinen einzelne Handschriften gegen andere sahidische Textzeugen des Johannesevangeliums korrigiert worden zu sein, was ebenfalls zur Kontamination der handschriftlichen Überlieferung beigetragen hat. Ferner hat die neutestamentliche Textüberlieferung - verglichen mit anderen aus der Alten Kirche überlieferten Texten - eine sehr hohe Stabilität, sodass, trotz der hohen Zahl der Handschriften, die Abweichungen relativ gering ausfallen. ${ }^{36}$ Das Resultat dieser Überlieferungssituation und der Besonderheiten der neutestamentlichen Textüberlieferung ist, dass für die sahidische Überlieferung des Johannesevangeliums ein eindeutiges Stemma nur schwer möglich ist. ${ }^{37}$ Hier ist das Verständnis der Textüberlieferung noch bei Weitem nicht so weit fortgeschritten wie bei der Editio Critica Maior. ${ }^{38}$ Dies wird auch noch einmal durch die Arbeit von Christian Askeland bestätigt. Dieser hat vor wenigen Jahren die Diskussion um die Textgeschichte der sahidischen Version des Johannesevangeliums aufgegriffen und mit guten Argumenten die von Schüssler aufgestellte These eines besonders hohen Alters von sa 1 zurückgewiesen. Das

closely as possible. As no other text it sticks to the Greek 'Vorlage' often in almost slavish imitation. sa 561 provides the most ancient form of text.“

35 Siehe hierzu auch Askeland 2012: 94: „James Barr argued that ancient translation traditions generally became more formally literal over time, redacting their versions to replicate their theoretical source texts more strictly and less idiomatically.“

36 Es sei auch nur auf die im Vergleich dazu sehr großen Textunterschiede der verschiedenen Zeugen des Apokryphons des Johannes hingewiesen; siehe hierzu Förster 2017: 504-505 und die dort angegebene Literatur.

37 Siehe hierzu auch Maas 1960: 8: „Wenn die erste der in $\S 6$ genannten Voraussetzungen nicht zutrifft, wenn also einzelne Schreiber mehrere Vorlagen kontaminiert haben, so ist in dem Bereich dieser Kontaminationen die eliminatio stark behindert, wenn nicht unmöglich. Die Kontamination verrät sich daran, daß der kontaminierte Zeuge einerseits Sonderfehler seiner eigenen Vorlage nicht zeigt, weil er aus einer anderen das Richtige entnommen hat, andererseits Sonderfehler solcher Vorlagen zeigt, von denen er in der Hauptsache nicht abhängt.“

38 Allerdings besteht auch dort nicht immer Sicherheit über den Ausgangstext; vgl. Wachtel 2017: $12^{\star}-17^{\star}$, dort $12^{\star}:$, „...] an 155 Stellen haben die Herausgeber die Entscheidung über den Ausgangstext offen gelassen und die infrage kommenden Varianten untereinander in die Leitzeile gesetzt.“ 
erste zusätzliche Problem, auf das Askeland hinweist, ist dabei die Tatsache, dass zahlreiche der Handschriften zu fragmentarisch sind, um sie (auch wegen der im Vergleich mit anderen antiken Texten geringen Textabweichungen) tatsächlich in jedem Fall sicher einer bestimmten Textgruppe zuordnen zu können. ${ }^{39}$ Ferner ist Askeland zuzustimmen, dass das Alter einer Handschrift in vielen Fällen nur wenig über das Alter der in ihr bezeugten Version des biblischen Textes aussagt. ${ }^{40}$

An einzelnen Stellen wird in der vorliegenden Edition ein Vers wiederholt. Dies geschieht dann, wenn sich die handschriftliche Überlieferung dahingehend aufteilt, dass kombinierte Varianten von Handschriftengruppen bezeugt werden. In solchen Fällen würde die Verzeichnung der Einzelvarianten im kritischen Apparat die Kombination mehrerer Varianten in einer handschriftlichen Überlieferung verdecken (vgl. Joh 1,39). In diesem Sinn darf der Zugang als „minimalistisch“ bezeichnet werden. ${ }^{41}$

Für die vorliegende Edition wurde auch die Interpunktion des Sahidischen regularisiert. Erkenntnisse bezüglich der Bedeutung einzelner Aspekte der Interpunktion hat Matthias H. O. Schulz in Anhang 2 zusammengestellt. Es war im Rahmen der technischen Möglichkeiten schlicht unmöglich, die Interpunktion aller einzelnen Handschriften mit allen Varianten bei der Edition bzw. in einem kritischen Apparat zu berücksichtigen. ${ }^{42}$ Dass damit eine Edition möglicherweise den eigentümlichen Charakter einer Handschrift verliert, wie dies Karlheinz

39 Askeland 2012: 95: „In order to establish trends, the assessment must prioritize complete, continuous manuscripts.“

40 Askeland 2012: 94: „The current discussion will problematize Schüssler’s hypothesis with respect to two of his presuppositions. First, the author has precariously oversimplified the dating of his witness; $\mathrm{sa}^{1}$ is assumed to be comparatively early and $\mathrm{sa}^{4.5 .9}$ are presumed to be comparatively late. Not only are the dates of these manuscripts uncertain, but, additionally, temporal priority is not equivalent to generic priority. A late manuscript could preserve an early text. Second, Schüssler has not presented any evidence contradicting his thesis - and such evidence does exist.“

41 Askeland 2012: 107: „[...] research should include the most data possible without contaminating the data set with ambiguous or irrelevant information. The tiny fraction of the manuscript tradition which is extant may not be representative of the lost tradition, and explorations of the data must ruthlessly avoid over-simplification.“

42 Dies geschieht in Absetzung zu Karlheinz Schüsslers Forderungen, wie eine kritische Edition eines sahidischen Bibeltextes auszusehen hat; vgl. Schüssler 2008: 61: „Only when seeing the original text in front of us with all its mistakes, additions, supplements, and corrections may we be able to sense what the interpreter left in his day to the receiver of his gift or to his customer by his work.“ 
Schüssler postuliert, ${ }^{43}$ war nicht $\mathrm{zu}$ verhindern. Es mag, um es mit Karlheinz Schüssler zu formulieren, ein „unpersönlicher“ Text geworden sein, es ist jedoch der Versuch, einen verlässlichen Text mit allen graphischen und textlichen Varianten zu bieten. Diese werden - mit den im Nestle/Aland gebrauchten textkritischen Zeichen - wie auch bei Horner in zwei getrennten kritischen Apparaten geboten. Die hohe Zahl der fragmentarisch erhaltenen Handschriften hat zur Folge, dass - im Gegensatz zu Horner - die Auflistung der einzelnen Zeugen, die für einen Vers angeführt werden, nicht im kritischen Apparat auf der jeweiligen Seite geboten wird, sondern separat als Anhang geführt wird. Ferner werden alle zitierten Handschriften in einer eigenen Liste mit den für die Edition zentralen Informationen dargeboten (vgl. die Übersicht über die aufgenommenen Handschriften). Für weiterführende Informationen wie die BCNummern, Aufbewahrungsorte der einzelnen Blätter einer Handschrift und ähnliche Informationen kann auf die SMR-Datenbank verwiesen werden. Ferner kommt es in der Koptologie regelmäßig vor, dass zusätzliche Blätter einzelner Handschriften identifiziert werden. Dies wird in der SMR-Datenbank vermerkt. Für die Frage, ob alle Blätter einer Handschrift der Edition zugrunde gelegt werden konnten oder ob nach Veröffentlichung der Edition noch weitere Blätter gefunden wurden, sei deswegen ebenfalls auf die SMR-Datenbank verwiesen, die kontinuierlich aktualisiert wird, was bei einem publizierten Buch nicht möglich ist. ${ }^{44}$

Abschließend darf somit festgehalten werden, dass die hier vorgelegte Edition die erste moderne kritische Edition eines Evangeliums im sahidischen Dialekt ist, die alle erreichbaren handschriftlichen Zeugen berücksichtigt. Die Aufnahme in der Fachwelt wird zeigen, ob die hier gewählte Editionsform und damit der Verzicht auf einen aus methodischer Sicht wohl zum Scheitern verurteilten Versuch, ${ }^{45} \mathrm{zu}$ diesem Zeitpunkt einen wie auch immer gearteten Arche-

43 Schüssler 2008: 41: „This 'spirit' that is met in the original manuscripts has entirely disappeared in our contemporary edition of the Bible. The text of the NA27 is impersonal and without character compared to a codex Vaticanus for instance. It might originally have read like that, surely different at some other places.“

$44 \mathrm{http}: / /$ intf.uni-muenster.de/smr/.

45 Für die Bedeutung der Fehler, die beim Abschreiben entstehen, zur Erstellung eines Stemmas sei auf Maas 1960: 26-30 (Abschnitt Leitfehler und stemmatische Archetypen), dort 26 verwiesen: „In der Lehre von den Abhängigkeitsverhältnissen der Handschriften - es sei gestattet, von 'Stemmatik' zu reden - haben die Fehler, die beim Abschreiben entstehen, entscheidende Bedeutung. “ Wenn nun Handschriften wiederholt gegeneinander und gegen griechische Ausgangstexte korrigiert werden, verlieren diese Fehler für die Bestimmung eines Archetypen an Bedeutung. 
typ zu konstruieren, Zustimmung finden wird. Damit teilt das sahidische Johannesevangelium das Schicksal zahlreicher Klassikerhandschriften. ${ }^{46}$ Die Alternative wäre der Verzicht auf eine kritische Edition der sahidischen Version des Johannesevangeliums gewesen.

Für die Überlieferung des griechischen Neuen Testaments, bei der es sich ebenfalls um eine hoch kontaminierte Textüberlieferung handelt, darf jedenfalls festgehalten werden, dass - dies zeigen die Änderungen des kritischen Textes in der Edition von Nestle/Aland - im 20. und 21. Jahrhundert zahlreiche Änderungen am präsumtiven Archetyp vorgenommen wurden.

\section{Die Anhänge}

Der Edition sind einige Anhänge beigefügt. Der erste Anhang besteht aus einer Auflistung der benutzten Handschriften und einer Auflistung der für jeden einzelnen Vers zitierten Handschriften (Anhang 1). Ein zweiter Anhang stellt wichtige Beobachtungen zu den Handschriften und ihren Paratexten zusammen, welche im kritischen Apparat keinen Platz gefunden hätten (Anhang 2). Da auch bei anderen Editionen koptischer Zeugen des Neuen Testaments auf eine Übersetzung des Texts verzichtet wurde - es sei auch nur auf die Texteditionen von Quecke oder Sharp verwiesen - müsste nicht eigens begründet werden, warum keine deutsche Übersetzung geboten wird.

Bei der sahidischen Version des Johannesevangeliums handelt es sich um die Übersetzung eines griechischen Textes. Die Verwendung des Begriffs „griechischer Text“ soll dabei keinesfalls eine Vorentscheidung über eine konkrete der Übersetzung zugrundeliegende griechische Textform darstellen, sondern den hier edierten Text als einen übersetzten Text ausweisen. Schließlich wird im Rahmen der neutestamentlichen Textkritik selbstverständlich die Frage aufgeworfen, welche konkrete griechische Textvariante einem in das Sahidische übersetzen Wort oder einer Phrase zugrunde gelegen haben dürfte. In diesem Zusammenhang lässt sich Folgendes festhalten: Die beim sahidischen Johannesevangelium verwendete Übersetzungstechnik war formal äquivalent, es handelte sich also um das, was man gemeinhin als „wortgetreue“ Übersetzung zu bezeichnen pflegt. Derartige Übersetzungen stellen jedoch gerade durch ihre formalen Aspekte eine große und wohl bisher unterschätzte Herausforderung

46 Siehe Maas 1960: 9: „Die Abhängigkeitsverhältnisse der Klassikerhandschriften sind großenteils noch nicht abschließend untersucht, auch abgesehen von den häufigen Fällen, wo Kontamination ein sauberes Ergebnis nicht erhoffen lässt.“ 
dar, wenn es darum geht, die Unterschiede gegenüber dem Ausgangstext in einer modernen Übersetzung herauszuarbeiten bzw. eine tatsächlich dem Text gerecht werdende Übersetzung zu erstellen. Horner, der in seiner Edition eine Übersetzung abgedruckt hatte, kann in diesem Kontext nur sehr bedingt als Vorbild für die hier vorgelegte Edition gelten, da seine Übersetzung sich sehr stark an eine traditionelle englische Übersetzung anlehnt. Wie sehr traditionelle Übersetzungen in den verschiedenen modernen Sprachen aufgrund der verwendeten Instrumente im Lateinischen verhaftet sind, ist Teil einer derzeit geführten Diskussion. ${ }^{47}$ Dies führt in letzter Konsequenz dazu, dass Übersetzungen in theologisch entscheidenden Fragen manchmal näher an der Vulgata als am griechischen Text sind. Schließlich scheint die grundsätzlich ebenfalls formal äquivalent übersetzende Vulgata weitaus stärker interpretierend zu übersetzen, als dies gemeinhin wahrgenommen wird..$^{48}$ Die fehlende Wahrnehmung dieser Problematik dürfte wahrscheinlich darin ihren Ursprung haben, dass formale Äquivalenz oftmals mit dem Konzept einer „wortgetreuen“ Übersetzung gleichgesetzt wird. Allerdings kann sich in formaler Äquivalenz große inhaltliche Distanz verbergen. Für die koptische Überlieferung des Johannesevangeliums konnte gezeigt werden, dass bereits kleine Übersetzungsentscheidungen des Koptischen, die im Einzelfall nur eine Konjunktion betreffen mögen, ${ }^{49}$ in entscheidender Weise das theologische Verständnis des Textes zu verändern geeignet sind.$^{50}$ Die Erstellung der oben erwähnten Anhänge war im konkreten Zeitrahmen möglich. Eine wissenschaftlich solide Untersuchung der semantischen Differenzen zwischen griechischem Ausgangstext und sahidischer Übersetzung, welche die theologischen Implikationen sowie potentielle mit dieser Übersetzung verbundene Identitätskonstruktionen der koptischen Kirche berücksichtigen müsste, hätte den vorgegebenen Zeitrahmen bei weitem gesprengt und kann möglicherweise Gegenstand weiterer Forschungsprojekte werden. Gleiches gilt für Indices der griechischen und koptischen Wörter, so sehr es sich dabei ebenfalls um ein Desiderat handeln mag. Angesichts des im Projekt vorgegebenen Zeitrahmens war es nötig, Prioritäten zu setzen. Ob diese Priorisierung zu Recht geschehen ist, mögen andere beurteilen. Allerdings wäre das hier vorgelegte Werk andernfalls überhaupt nicht erschienen.

Als weitere Anhänge sind der lykopolitanische und der proto-bohairische Text (Anhänge 3 und 4) im vorliegenden Band abgedruckt. Die Texte wurden im

47 Vgl. grundsätzlich Förster 2020a und Förster 2020b.

48 Siehe einleitend Förster 2019.

49 Vgl. Förster 2016.

50 Siehe Förster 2011b. 
Rahmen der Verwendung der koptischen Überlieferung für die Editio Critica Maior kollationiert. Für Proto-Bohairisch wurden die Edition von Kasser (Kas.) ${ }^{51}$, seine Korrekturen (Réex.) ${ }^{52}$ und Sharps Edition (Sha.) ${ }^{53}$ zugrunde gelegt und gegen Bilder der Handschrift abgeglichen, um einen möglichst guten Text zu bieten. Dabei sind gegenüber beiden Editionen und Kassers Korrekturen Verbesserungen möglich gewesen, doch wurden auch Lesungen bzw. Emendierungen (vor allem von Kasser) berücksichtigt, die anhand der Handschriftenbilder nicht immer nachvollziehbar sind. Abweichungen zu Editionen und Korrekturen sind in einem Apparat verzeichnet. Für die Interpunktion wurde weitgehend Kassers editio princeps zugrundegelegt, da Sharp statt Interpunktion gewöhnlich nur Spatien verzeichnet, doch gesetzte Punkte nicht angibt.

Der lykopolitanische Text folgt den Editionen von Thompson (ly 1$)^{54}$ sowie Funk und Smith (ly 2) ${ }^{55}$. Auch sie wurden gegen Bilder der Handschriften abgeglichen, um einen möglichst guten Text $\mathrm{zu}$ bieten. Abweichungen $\mathrm{zu}$ Editionen sowie abweichende Lesungen von ly $2 \mathrm{zu}$ ly 1 sind ebenso in einem eigenen Apparat verzeichnet. Für ly 1 waren vorhandene schwarz-weiß-Abbildungen zwar geeignet, um Lesungen von Buchstaben zu überprüfen, doch nicht um die Papyrusstruktur von Setzungen der Supralinearstriche und der meisten Interpunktionszeichen sicher zu unterscheiden. Daher wurde für den lykopolitanischen Text weitgehend darauf verzichet, Diakritika und Interpunktion zu verzeichnen. Hier bleibt Thompsons Edition weiterhin autoritativ.

Ohnehin haben Diakritika und Interpunktion für den intendierten Sinn der Anhänge eine untergeordnete Bedeutung. Beide Anhänge sollen einen komfortablen Zugang zu zwei der wichtigsten kleineren koptischen Dialektversionen des Johannesevangeliums bieten, um sie für Direktvergleiche mit dem sahidischen Text heranziehen zu können. Es ist nicht Aufgabe dieser Anhänge, bestehende Editionen zu ersetzen. Gleichzeitig ist damit für jeden, der mit der sahidischen Überlieferung des Johannesevangeliums arbeitet, eine Ausgabe geschaffen, die ein konkordantes Arbeiten mit den anderen frühen dialektalen Zeugen des Johannesevangeliums ermöglicht.

Die bohairische Überlieferung bedarf dringlich einer eigenen kritischen Edition $^{56}$ und wurde deshalb nicht abgedruckt. Auch wurde auf einen Abdruck der

51 Kasser 1958 [Kas.].

52 Kasser 2001 [Réex.].

53 Sharp 2016 [Sha.].

54 Thompson 1924 [ly 1; Verweise auf die Edition erfolgen durch Tho.].

55 Funk/Smith 1990 [ly 2].

56 Förster 2020. 
extrem fragmentarischen Zeugen des Johannesevangeliums in anderen kleinen Dialekten verzichtet. Diese sind dank der Arbeit von Christian Askeland bereits gut über eine Datenbank zugänglich. ${ }^{57}$

57 Vgl. http://www.iohannes.com/transcriptions/index.html?witness=ac1\&language=coptic. 